\title{
POLÍTICAS PÚBLICAS DE SAÚDE Tendências recentes
}

\author{
Mariana Vieira da Silva \\ Instituto Universitário de Lisboa (ISCTE-IUL), CIES-IUL, Lisboa, Portugal
}

\section{Introdução}

As políticas de saúde são centrais para a análise do estado-providência contemporâneo: não apenas representam uma das principais parcelas das despesas sociais, a par das pensões, como têm implicações universais e ao longo de todo o ciclo de vida. Além disso, sobre os sistemas de saúde contemporâneos pendem tensões e pressões semelhantes às que encontramos noutras áreas sociais: aumento continuado da despesa, pressões demográficas, arrefecimento do crescimento económico e constrangimentos fiscais.

No entanto, a área da saúde tem especificidades importantes: a complexidade económico-financeira que decorre em grande parte da inovação tecnológica e terapêutica e a centralidade do poder dos profissionais de saúde. Destas especificidades resulta um conjunto de desafios à mudança nas políticas.

Neste texto procurar-se-á descrever as principais tendências na evolução do sistema de saúde em Portugal, a partir do campo das políticas públicas, identificando as principais fases e tendências de mudança, bem como os problemas e desafios mais relevantes que atualmente se colocam.

\section{O agendamento do problema da contenção de custos}

As mudanças nas políticas de saúde aceleraram a partir da década de 1980 em toda a Europa, com o objetivo explícito fundamental de conter o crescimento da despesa de forma a garantir a sustentabilidade dos sistemas de saúde. A essência do puzzle nesta área das políticas públicas nas últimas décadas, mesmo em sistemas de diferentes tipos (Wendt, 2009), centra-se na redefinição do papel do estado no financiamento, no pagamento, na regulação e na prestação de cuidados de saúde (Saltman, 1994).

Com as necessárias diferenças de contexto, as mudanças podem ser resumidas (quadro 1) na evolução para um sistema de saúde mais híbrido (entre o modelo beveridgiano e o modelo bismarckiano, que estiveram na génese dos sistema de proteção social na maior parte dos países desenvolvidos). “Em diversos países a estratégia seguida foi no sentido da combinação de elementos dos dois modelos. Procurou-se uma utilização plural de incentivos típicos do mercado, com a manutenção da propriedade pelo setor público" (Simões, 2004: 64).

Assim, conforme pode ser observado no quadro 1, os países com serviços nacionais de saúde semelhantes ao português - sistemas de saúde universais, financiados através dos impostos e em que o estado tem um papel relevante na prestação 
Quadro 1 Tipos de sistema de saúde $\left({ }^{*}\right)$ e estratégias de mudança

\begin{tabular}{l|l|l}
\hline Tipos de sistema de saúde & Objetivos da mudança & Exemplos de políticas \\
\hline $\begin{array}{l}\text { Países sem seguro/cobertura } \\
\text { universal (EUA) }\end{array}$ & $\begin{array}{l}\text { Esforços para garantir a } \\
\text { universalização da cobertura }\end{array}$ & $\begin{array}{l}\text { Reforma "Obama" } \\
\text { Universalização }\end{array}$ \\
\hline $\begin{array}{l}\text { Países com seguros } \\
\text { sociais/seguros universais (França, } \\
\text { Alemanha) }\end{array}$ & $\begin{array}{l}\text { Alargar fontes de financiamento } \\
\text { estão incluídos no mercado de } \\
\text { trabalho }\end{array}$ & $\begin{array}{l}\text { Alemanha: os cuidados com as } \\
\text { crianças passaram a ser pagos por } \\
\text { impostos }\end{array}$ \\
\hline $\begin{array}{l}\text { França: uma taxa adicional sobre } \\
\text { salários mais elevados com efeito } \\
\text { redistributivo }\end{array}$ \\
$\begin{array}{l}\text { Países com serviço nacional de } \\
\text { saúde (UK, Suécia, Portugal, } \\
\text { Espanha) }\end{array}$ & $\begin{array}{l}\text { Separação entre os papéis de } \\
\text { financiador, regulador e prestador } \\
\text { Concorrência entre setor público e } \\
\text { privado } \\
\text { Remercadorização }\end{array}$ & $\begin{array}{l}\text { Hospitais geridos por privados } \\
\text { Adoção de mecanismos de } \\
\text { mercado } \\
\text { Privatização de parte dos cuidados } \\
\text { de saúde }\end{array}$ \\
\hline
\end{tabular}

* Retomamos aqui a tipologia utilizada pela OCDE em 1987, assente na propriedade, financiamento e cobertura dos sistemas de saúde.

dos cuidados (Inglaterra, Suécia, Espanha, Finlândia, Espanha e Itália) - prosseguiram políticas semelhantes para enfrentar as pressões de (in)sustentabilidade.

A disseminação de modelos de gestão típicos do mercado e separação dos vários papéis desempenhados pelo estado (regulação, financiamento e prestação de cuidados) foi um dos elementos fundamentais desta estratégia. Mercado interno, competição pública ou quasi-mercado são alguns dos conceitos explorados por vários autores para descrever este tipo de mudanças (Le Grand e Bartlett, 1993; Saltman e Van Otter, 1989).

Neste sentido, podem identificar-se, recorrendo à proposta do Observatório Português dos Sistemas de Saúde (Relatório da Primavera), de Carapinheiro e Pinto (1986) e de Campos (2011), seis grandes fases do Serviço Nacional de Saúde (SNS) português:

a) 1970 a 1982, um período de criação e expansão do SNS;

b) 1982 a 1995, em que a agenda predominante é a mudança da fronteira entre público e privado;

c) 1995 a 2002, em que a agenda predominante é a do aumento dos serviços existentes e em que é visível o recuo na narrativa da privatização do SNS, desenvolvendo, todavia, as políticas de criação de um mercado interno;

d) 2002 a 2005, em que foi visível uma combinação de estratégias centradas na eficiência do sistema com um discurso de mudança para um sistema misto entre público e privado e de substituição do Serviço Nacional de Saúde por um Sistema Nacional de Saúde; 
Quadro 2 Instrumentos de reforma em Portugal

\begin{tabular}{l|l}
\hline $\begin{array}{l}\text { Separação entre financiamento e prestação dos } \\
\text { cuidados }\end{array}$ & $\begin{array}{l}\text { Contratualização entre estado e hospitais públicos } \\
\text { Gestão por objetivos }\end{array}$ \\
\hline $\begin{array}{l}\text { Competição entre hospitais públicos e entre hospitais } \\
\text { públicos e privados - mercado interno }\end{array}$ & $\begin{array}{l}\text { Hospitais privados incluídos na rede pública, } \\
\text { Amadora-Sintra } \\
\text { Deduções fiscais } \\
\text { Sistema remuneratório }\end{array}$ \\
\hline Diminuição do papel do estado enquanto prestador & $\begin{array}{l}\text { Acordos de cirurgia concessionada } \\
\text { Alargamento aos privados de novas áreas de } \\
\text { continuado de cuidados (como os cuidados a medicina oral através do cheque } \\
\text { dentista) } \\
\text { Parcerias público-privadas }\end{array}$ \\
\hline Contenção de custos & $\begin{array}{l}\text { Diminuição das comparticipações em medicamentos } \\
\text { Aumento das taxas moderadoras, limitação da } \\
\text { utilização de meios complementares de diagnóstico } \\
\text { Prevenção } \\
\text { Incentivo aos medicamentos genéricos }\end{array}$ \\
\hline
\end{tabular}

e) 2005 a 2009, com políticas que acentuam a manutenção da referência ideológica do SNS e alargamento e modernização de serviços, ao mesmo tempo que se mantêm e acentuam as estratégias que visam os ganhos de eficiência;

f) desde 2010, com a aplicação de medidas de austeridade impostas pelas revisões do Programa de Estabilidade e Crescimento e pelas obrigações do estado português no âmbito do Plano de Assistência Financeira.

À luz destas fases podemos reconhecer um conjunto de fatores de continuidade, e alguns elementos de mudança no SNS. Apesar da evolução no sentido da diminuição da intervenção direta do estado, que aconteceu em Portugal, como os restantes países com SNS, mantém-se um sistema público, financiado por impostos, que funciona como seguro público, universal e obrigatório. O estado permanece, também, prestador de serviços de saúde e proprietário de uma parcela significativa do sistema.

Mas podemos, igualmente, identificar importantes elementos de mudança, em linha com as estratégias descritas no quadro 2: a separação entre financiamento e prestação dos cuidados, recorrendo nomeadamente à contratualização entre estado e hospitais públicos; a criação de um mercado interno regulado pelo estado, garantindo competição entre prestadores públicos e entre prestadores públicos e privados; alterações do sistema remuneratório dos médicos, afastando-os do regime de funcionário público (salários) e aproximando-o de profissionais liberais (honorários, diversificação salários), ou a valorização dos cuidados de saúde privados ou concessionados, nomeadamente para responder às listas de espera. 


\section{Três objetivos das políticas de saúde: sustentabilidade; equidade; resultados e qualidade do sistema}

As últimas décadas demonstram uma evolução nas mudanças nas políticas de saúde. $\mathrm{O}$ trabalho de diversas instituições internacionais (OCDE, Banco Mundial, Organização Mundial de Saúde e União Europeia) marca definitivamente um olhar abrangente sobre aos sistemas de saúde, que influencia de forma indelével a formulação e implementação de políticas. Os relatórios publicados por estas instituições estabeleceram para esta área das políticas públicas parâmetros de avaliação dos sistemas centrados simultaneamente na sua sustentabilidade e na eficiência, no acesso e equidade dos modelos de prestação de cuidados de saúde e na qualidade dos serviços prestados.

No fundo, estes parâmetros tornaram-se um referencial, não apenas para aferir e comparar mas também para promover as mudanças nos sistemas e nas políticas. Tal quadro analítico e comparativo mais amplo permite ir além das discussões centradas apenas nos aspetos parcelares da gestão e discussão política de cada um dos sistemas: contenção de custos, relações estado-mercado, etc. A partir deste quadro mais alargado, podemos, pois, estudar as mudanças nas políticas de saúde e avaliar os sistemas de saúde em torno de três grandes finalidades: (a) sustentabilidade e eficiência financeira; (b) equidade no acesso; e (c) qualidade dos cuidados e estado de saúde das populações.

\section{Sustentabilidade do sistema}

Uma das finalidades chave das políticas de saúde é a garantia da sustentabilidade do sistema, que decorre genericamente do problema do crescimento continuado da despesa (que em Portugal tinha, em 2009, ultrapassado os 10\% do PIB).

No caso específico da saúde, o crescimento continuado da despesa que ocorre na generalidade das políticas sociais e que, aliado ao abrandamento do crescimento económico, constitui um dos principais problemas políticos, é agravado por três elementos essenciais. O primeiro fator prende-se com os custos que decorrem de uma maior procura de cuidados de saúde, dos recursos humanos e da crescente especialização das profissões médicas e paramédicas. O segundo fator relaciona-se com o peso crescente das tecnologias em saúde e o alargamento substancial das suas utilizações. O peso que este setor em particular tem resulta não só de uma pressão financeira, mas também de uma pressão económica resultante da proximidade entre o setor farmacêutico e o setor financeiro. $O$ terceiro prende-se com uma composição da despesa mais exigente, ligada à cada vez maior relevância das despesas associadas à evolução demográfica e, em particular, ao acentuado aumento da esperança de vida.

\section{Universalidade e equidade no acesso}

Um dos objetivos fundamentais para os sistemas de saúde é a equidade. Podemos enquadrar neste objetivo duas dimensões: a universalidade do sistema (todos 
devem ter acesso) e a igualdade perante o tratamento independentemente da condição financeira (ou seja, a qualidade do tratamento não deve depender dos recursos financeiros, mas sim da necessidade do mesmo tratamento).

Portugal tem inscrito na Constituição um sistema de saúde universal (que abrange todas as pessoas), geral (que abrange todos os tratamentos necessários) e tendencialmente gratuito (em que os cuidados não são sujeitos a pagamento, apenas são possíveis taxas que moderem o recurso ao sistema). É um sistema com elevada equidade, no sentido em que a lei protege todos os cidadãos de igual forma, num sistema de saúde em que cada um contribui (através dos impostos) no âmbito das suas possibilidades para beneficiar de todas as potencialidades do sistema de acordo com as suas necessidades.

No entanto, persistem desigualdades no acesso (Barros e Simões, 2007). Por um lado, áreas com pouca cobertura (por exemplo, os cuidados dentários), por outro, áreas em que o peso de um sistema privado complementar é significativo e em que o sistema público dá resposta mais limitada (por exemplo, as consultas de algumas especialidades).

\section{Qualidade dos cuidados e o estado de saúde das populações}

Um dos elementos centrais do contributo das instituições internacionais para as mudanças políticas na saúde prende-se com a valorização da qualidade dos cuidados e do estado de saúde das populações. De facto, são hoje praticamente inexistentes projetos de mudança política que não incorporem conceitos e indicadores que vão além da dimensão da sustentabilidade financeira dos sistemas. Por exemplo, impactos na esperança média de vida e taxas de mortalidade infantil, de cobertura de tratamentos e meios de diagnóstico, tempos e listas de espera, ou a prevalência de algumas patologias, entre outros elementos.

Aliás, de tal forma a questão da qualidade e dos cuidados se tornou central para a avaliação e gestão dos sistemas de saúde que, neste campo, de forma excecional no panorama das políticas públicas, se sedimentou a ideia de que as mais inovadoras tecnologias e os mais recentes saberes profissionais devem estar disponíveis para todos a um custo comportável, como aliás a própria OMS reconheceu na Declaração de Alma-Ata, em 1978 (Sakellarides, 2001: 102).

A qualidade dos cuidados funciona como polo agregador deste triplo objetivo que, na verdade, forma um triângulo: o sistema só tem qualidade se tiver garantido a sustentabilidade e a equidade; só pode ser equitativo se for sustentável e tiver a mesma qualidade para todos; e só pode ser sustentável se tiver qualidade distribuída de forma equitativa. É a combinação destes três fatores que tem limitado as alterações estruturais e tem garantido a legitimidade política e social do SNS. 
Dois desafios a superar

a) As tecnologias e as funções do estado em saúde

A saúde tem um nível maior de complexidade que as restantes áreas das políticas públicas normalmente incluídas no campo mais amplo do estado de bem-estar. É verdade que existem inúmeras características comuns a outras áreas do estado-providência, e que sobre os sistemas de saúde impendem as mesmas pressões (nomeadamente a questão demográfica), mas existem especificidades que se constituem como constrangimentos específicos às políticas de saúde. A principal característica distintiva da área da saúde assenta na sua profunda articulação com setores económicos e nas consequências desta articulação para as funções do estado na regulação dos diversos produtores de cuidados (Moran, 1995 e 2000).

A qualidade e a eficácia dos cuidados de saúde depende fortemente da dinâmica económica de atividades industriais e de serviços muito competitivas e, frequentemente, muito globalizadas (indústria farmacêutica, setor dos equipamentos médicos ou a comercialização de fármacos). Por outro lado, alguns desses atores económicos desenvolvem articulações estratégicas com os prestadores de cuidados no plano da investigação e desenvolvimento, o qual, por sua vez, influencia e é influenciado pela natureza e qualidade dos sistemas de ensino e formação.

A rentabilização de investimentos complexos e de grande dimensão, a pressão de mercados concorrenciais multifacetados, e onde não é rara a existência de fenómenos de concentração do poder de mercado, fazem da complexidade da envolvente económica do setor da saúde um fator determinante para o seu desenvolvimento.

Desta forma, a superação da tensão entre a necessidade de controlar a evolução da despesa e a vontade de melhorar a eficácia clínica dos atos médicos e de dinamizar a própria economia torna-se muito exigente e solicita uma difícil articulação entre as funções de financiamento, de prestação de cuidados e de regulação dos interesses económicos que o estado é chamado a desempenhar. O facto de os interesses do estado no desempenho destas funções serem, por vezes, contraditórios constitui um dos desafios mais relevantes das políticas públicas de saúde.

b) A necessidade de novas coligações: a autonomia clínica e as políticas de saúde

A concretização de políticas que combinem a garantia da sustentabilidade dos sistemas de saúde com o aumento da equidade dos modelos de prestação de cuidados e com a sua qualidade depende não só de coligações políticas e sociais, mas também da participação dos profissionais de saúde nas medidas de contenção de custos e de ganhos de eficiência.

As estratégias de ganho de eficiência assentaram nas últimas décadas em áreas como a alteração dos modelos de administração (empresarialização, nova gestão pública), na otimização dos procedimentos de gestão (por exemplo, centrais de compras, externalização de serviços) e em correções de ineficiências de rede (por exemplo, encerramento de unidades de pequena dimensão, centralização de serviços). Sendo 
certo que haverá sempre margem para mais progressos, estar-se-á perto de um ponto em que os ganhos a este nível (gestão, rede) serão insuficientes para produzir mais impactos significativos na promoção da sustentabilidade do sistema.

O lugar central que os profissionais de saúde ocupam em qualquer estratégia de mudança nas políticas explica-se olhando para quatro esferas do seu poder profissional: o poder económico (assente não só na propriedade dos lugares de produção dos cuidados de saúde, como na definição dos honorários e nas condições em que os serviços são prestados - quem tratar, como tratar, quando tratar); o poder estratégico (capacidade de influenciar as políticas e de as legitimar, mas também de definir os objetivos de trabalho para os profissionais); o poder administrativo (poder de gestão nos hospitais, direções clínicas, etc.); e, principalmente, o poder operacional (poder que resulta da autonomia clínica, do julgamento clínico e da definição dos protocolos e dos procedimentos).

Assumir plenamente a autonomia clínica dos profissionais, ao mesmo tempo que reconhecemos que é na sua esfera de decisão que se definem as escolhas com impacto económico e financeiro, implica que estes têm de ser parte ativa - e aliás, decisiva - na definição de protocolos clínicos e de utilização eficaz e racionalizada dos meios disponíveis, de modo a favorecer condições de sustentabilidade de longo prazo, que de outro modo serão inviáveis.

\section{Conclusão}

No plano do debate político é frequente a oposição de modelos alternativos de cuidados de saúde apresentadas como capazes de superar os problemas de sustentabilidade financeira, de eficiência e de equidade e qualidade do sistema. Este tipo de mudanças políticas é descrito como mudanças big bang que, alterando a equação que define as responsabilidades públicas no financiamento, propriedade e prestação dos cuidados de saúde, solucionariam os principais pontos críticos.

Porém, o peso da dependência de percurso (Pierson, 1995) e os custos de transição contribuem para que a mudança nas políticas tenda a ser feita dentro do modelo existente em cada país, assentando em políticas incrementais. Por outro lado, o trabalho comparativo de sistemas de saúde desenvolvido pelas organizações internacionais contribuiu não só para que as decisões políticas sejam mais baseadas em evidências e opções concretizáveis, como para a crescente conviç̧ão de que não existe um modelo de sistema de saúde isento de problemas de sustentabilidade, equidade ou qualidade, capaz de que superar os desafios com que os diversos países se deparam.

No entanto, esta mudança não parece ter ocorrido no debate público. As tensões no debate político muitas vezes decorrem no plano da discussão em torno de modelos teóricos alternativos e não no plano das escolhas políticas possíveis nos quadros institucionais e políticos existentes. Estas disputas, que pela sua natureza tendem a ser extremadas, diminuem o espaço para a produção de consensos, ao mesmo tempo que retiram legitimidade ao sistema (sob crítica) e às próprias mudanças (que, incrementais, nunca correspondem na totalidade às expectativas criadas). 
O contraste entre os termos do debate político e as possibilidades reais de reforma, que aliás poderia constituir, por si, um obstáculo a superar (embora de tipo diferente dos acima desenvolvidos) é mais um elemento demonstrativo da importância do estudo e da investigação em políticas públicas.

\section{Referências bibliográficas}

Barros, Pedro P., e Jorge Simões (2007), “Portugal: health system review”, Health Systems in Transition, 9 (5): 1-140.

Campos, António C. de (2011), O Percurso da Saúde: Portugal na Europa, Coimbra, Almedina.

Carapinheiro, Graça, e Margarida P. Pinto (1986), “Políticas de saúde num país em mudança: Portugal nos anos 70 e 80", Sociologia, Problemas e Práticas, 1, pp. 71-109.

Le Grand, Julian, e Will Bartlett (1993), Quasi-Markets and Social Policy, Londres, Macmillan.

Moran, Michael (1995), "Three faces of the health care state", Journal of Health Politics, 20 (3), pp. 767-781.

Moran, Michael (2000), "Understanding the welfare state: the case of health care", The British Journal of Politics \& International Relations, 2 (2), pp. 135-160.

Pierson, Paul (1995), Dismantling the Welfare State? Reagan, Thatcher and the Politics of Retrenchment, Cambridge, Cambridge University Press, Cambridge Studies in Comparative Politics.

Sakellarides, Constantino (2001), “De Alma-Ata a Harry Potter: um testemunho pessoal", Cuidados de Saúde Primários, 2, pp. 101-108.

Saltman, Richard B. (1994), "A conceptual overview of recent health care reforms", European Journal of Public Health, 4 (4), pp. 287-293.

Saltman, Richard B., e Casten Von Otter (1989), “Public competition versus mixed markets: an analytic comparison!", Health Policy, 11 (1), pp. 43-55.

Simões, Jorge (2004), Retrato Político da Saúde. Dependência do Percurso e Inovação em Saúde. Da Ideologia ao Desempenho, Coimbra, Almedina.

Wendt, Claus (2009), "Mapping European healthcare systems: a comparative analysis of financing, service provision and access to healthcare", Journal of European Social Policy, 19 (5), pp. 432-445.

Mariana Vieira da Silva. Instituto Universitário de Lisboa (ISCTE-IUL), CIES-IUL. E-mail: marianagvs@gmail.com 